\title{
JET Experiments to Assess the Clamping of the Fast Ion Energy Distribution during ICRF heating due to Finite Larmor Radius Effects
}

\author{
A. Salmi ${ }^{1}$, M.J. Mantsinen ${ }^{1}$, P. Beaumont ${ }^{2}$, P. de Vries ${ }^{2}$, L.-G. Eriksson ${ }^{3}$, C. Gowers ${ }^{2}$, \\ P. Helander ${ }^{2}$, M. Laxåback ${ }^{4}$, J.-M. Noterdaeme ${ }^{5,6}$, D. Testa $^{7}$ \\ and EFDA JET contributors * \\ ${ }^{1}$ Helsinki University of Technology, Association Euratom-Tekes, Finland \\ ${ }^{2}$ Euratom/UKAEA Fusion Association, Culham Science Centre, Abingdon, United \\ Kingdom \\ ${ }^{3}$ Association EURATOM-CEA, CEA/DSM/DRFC, CEA-Cadarache, St. Paul lez \\ Durance,France \\ ${ }^{4}$ Alfvén Laboratory, Association Euratom-VR, Stockholm, Sweden \\ ${ }^{5}$ Max-Planck IPP-EURATOM Assoziation, Garching, Germany \\ ${ }^{6}$ Gent University, Belgium \\ ${ }^{7}$ CRPP, Association Euratom-Confédération Suisse, EFPL, Lausanne, Switzerland \\ ${ }^{*}$ see annex of J Pamela et al., Fusion Energy 2002 (Proc. $19^{\text {th }}$ Int. Conf. Lyon, 2002) \\ IAEA, Vienna (2002).
}

\begin{abstract}
Experiments have been performed on the JET tokamak with $2^{\text {nd }}$ harmonic ion cyclotron resonance heating (ICRH) of hydrogen in deuterium plasmas to assess the role of finite Larmor radius (FLR) effects on the resonant ion distribution function. More specifically, the clamping of high-energy resonant particle distribution due to weak wave-particle interaction at high energy is studied. The distributions of ICRH heated hydrogen ions have been measured with a high-energy neutral particle analyser in the range of $0.29-1.1 \mathrm{MeV}$. By changing the electron density the energy $\mathrm{E}^{*}$, around which the wave-particle interaction becomes weak, is varied. The dependence of the ion distribution on $\mathrm{E}^{*}$ is experimentally observed for a number of discharges and FLR effects are clearly seen to affect the high energy tail shape. Experiments have been analysed with the combination of ICRH modelling codes PION and FIDO, including FLR effects, and good agreement with measurements have been found.
\end{abstract}

\section{INTRODUCTION}

One of the principal ICRF heating schemes foreseen for ITER is second harmonic heating of tritium $\omega \approx 2 \omega_{\text {cT. }}$. For this heating scheme, as for other harmonic ICRF schemes with $\omega \approx n \omega_{\mathrm{ci}}$ and $\mathrm{n}>1$, the absorption of wave power at the ion cyclotron resonance is a finite Larmor radius effect. Consequently, the wave absorption by the resonating ions is weak at low energies but increases strongly as the ratio of the ion Larmor radius, $\rho=v_{\perp} / \omega_{\text {ci }}$, to the perpendicular wavelength of the fast wave increases. Here, $v_{\perp}$ is the ion velocity component in the direction perpendicular to the background magnetic field. However, when ion Larmor radius further increases (corresponding typically $\mathrm{MeV}$ range) the absorption weakens again and effectively prevents particles from reaching higher velocities. To predict with confidence the performance of second harmonic heating of tritium, as well as to estimate the consequences of ICRH heated fast particles on plasma stability and on some diagnostics in ITER, it is important to have a good understanding of the resonant ion energy distribution. The information of the fast ion energy content or mean energy is 
not enough in all cases. Therefore, detailed investigations of second and higherharmonic scenarios and, in particular of finite Larmor radius effects, are required in present-day tokamaks. In this paper, we will present results from the JET tokamak that demonstrate the important role of finite Larmor radius (FLR) effects on the highenergy part of the ICRF-driven ion distribution.

It is not trivial to define experimental conditions to test in detail the role of the finite Larmor radius effects during ICRF heating. Naturally, the creation of a fast ion population when ICRF power is applied at a harmonic of the ion cyclotron frequency is itself a manifestation of the finite Larmor radius effects. However, there is seldom enough experimental data to draw conclusions on the exact nature of the ICRF absorption process including the influence of finite Larmor radius effects. Essential for this sort of study are the good confinement of the energetic ions as well as an accurate means of measuring their energy distribution at high energies.

The experiments reported in this paper continue an earlier work [1] on the JET tokamak where it was found that for second harmonic heating the number of particles at high-energy part of the distribution decreases strongly when the ion energy reaches $\mathrm{E}^{*}$, the expected location for weak wave-particle interaction. By comparing the measured energy distributions between fundamental and second harmonic heating scenarios, the rapid fall-off seen in the distribution for the second harmonic case could be attributed to FLR effects. This was an important experimental result because it showed that the FLR effects can influence the distribution function of the resonating ions. Furthermore, it indicated a possibility to assess directly the importance of finite Larmor radius effects during high-harmonic ICRF heating by measuring the ion distribution function for different $\mathrm{E}^{*}$, as has been done in the experiments reported in this paper.

This paper is organised as follows. In Section 2, we discuss ICRF physics relevant for the present experiments and discuss the interplay between the distribution function and RF diffusion coefficient. In Section 3, the experimental set-up and measurements are presented and in Section 4, computer simulations which take FLR effects into account are compared with the experimental measurements. Section 5 concentrates in discussing certain remaining issues like for instance the possibility of the existence of an adiabatic barrier. Finally in Section 6 follows the Summary.

\section{RELEVANT ICRF PHYSICS}

The distribution function $f$ for the ICRF resonant ions can be derived using the quasilinear Fokker-Planck equation

$$
\frac{\partial f}{\partial t}=\hat{C}(f)+\hat{Q}(f)
$$

where $\hat{C}$ is a collision operator and $\hat{Q}$ is a quasi-linear RF diffusion operator. In order to gain insight into the details of the perpendicular fast ion distribution, we use this equation in a simplified form:

$$
\frac{\partial f\left(v_{\perp}, t\right)}{\partial t} \approx \frac{1}{v_{\perp}} \frac{\partial}{\partial v_{\perp}}\left[-\alpha v_{\perp} f+\frac{1}{2} \frac{\partial}{\partial v_{\perp}}\left(\beta v_{\perp} f\right)+\frac{1}{4} \gamma f+D_{\mathrm{RF}} v_{\perp} \frac{\partial f}{\partial v_{\perp}}\right]
$$

The equation is the same as in Ref. 2 with the exception that the RF diffusion coefficient, here denoted with $\mathrm{D}_{\mathrm{RF}}$, is not an expansion but fully includes finite orbit 
effects and is thus suitable for this work where $2^{\text {nd }}$ harmonic heating is studied. Here $f\left(v_{\perp}\right)=\int f(v) d v_{\|}$and $\alpha, \beta$ and $\gamma$ are the Spitzer collision coefficients [3] and $D_{R F}$ is given by

$$
D_{\mathrm{RF}}=K\left|\mathrm{~J}_{\mathrm{n}-1}\left(\frac{k_{\perp} v_{\perp}}{\omega_{\mathrm{ci}}}\right)+\frac{E_{-}}{E_{+}} \mathrm{J}_{\mathrm{n}+1}\left(\frac{k_{\perp} v_{\perp}}{\omega_{\mathrm{ci}}}\right)\right|^{2},
$$

where $\mathrm{J}_{\mathrm{n}}$ denotes the $n^{\text {th }}$ order of Bessel function of first kind, $n$ is the number of the cyclotron harmonic, $\mathrm{K}$ is a constant proportional to the square of the co-rotating electric field $E_{+}$and $\mathrm{k}_{\perp}$ and $\omega_{\mathrm{ci}}$ are the perpendicular wave number and the ion cyclotron frequency, respectively. Note, that in deriving of Eq. (1) it has been assumed [2] that $\mathrm{v}_{\perp} \gg \mathrm{v}_{\|}$and $\mathrm{v}>>\mathrm{v}_{\text {th }}$. In simulations we found out that typically the kinetic energy content in the perpendicular component of fast ions is 8-10 times higher than the energy in the parallel component thereby increasing the confidence in these approximations.

The steady-state solution for a test particle distribution is readily solved from Eq. (1) by integration and yields

$$
f\left(v_{\perp}\right) \approx f_{0} \exp \left(-\int_{0}^{v_{\perp}} d v_{\perp} \frac{-4 \alpha v_{\perp}+2\left(\beta v_{\perp}\right)^{\prime}+\gamma}{2 \beta v_{\perp}+4 D_{\mathrm{RF}} v_{\perp}}\right) .
$$

The above equation is the same as in [2] except for the term $D_{R F}$ which we have kept here unexpanded. To our aim it is not necessary to simplify the equation further. We only wish to point out that except close to the local minima of $D_{R F}$ the term " $4 D_{R F} v_{\perp}$ " is large compared to all other terms in the integrand for a wide parameter range (applies when $\mathrm{v}>>\mathrm{v}_{\mathrm{th}}$ ). In fact, if " $4 D_{R F} v_{\perp}$ " would be small no fast tail would be able to develop in the first place. After realising this, the qualitative behaviour of the distribution function, as shown in the subsequent figures, is easy to see; when the dominating term $\mathrm{D}_{\mathrm{RF}}$ is large the integrand is small and therefore the distribution stays flat, whereas when $D_{R F}$ is small the integrand is large and the distribution drops quickly.

Fig. 1(a) shows $D_{R F}$ for the fundamental (full line) and the second harmonic resonance heating (dash-dotted line) where FLR effects are included. In comparison, also an imaginary case where $\mathrm{D}_{\mathrm{RF}}$ is set to a constant is shown (dashed line). In Fig. 1(b) the corresponding distributions are plotted. The simple arguments given above are seen to be valid as the distribution functions behave as expected. In practise, if the first minimum of $\mathrm{D}_{\mathrm{RF}}$ in the plasma is small and wide enough then practically no particles can get accelerated beyond energy $E^{*}$ (the energy where $D_{R F}$ has its first minimum). Note that the power levels here are not equal. The purpose of the figure is to illustrate the drops in the distributions due to the weak wave-particle interaction regions. 

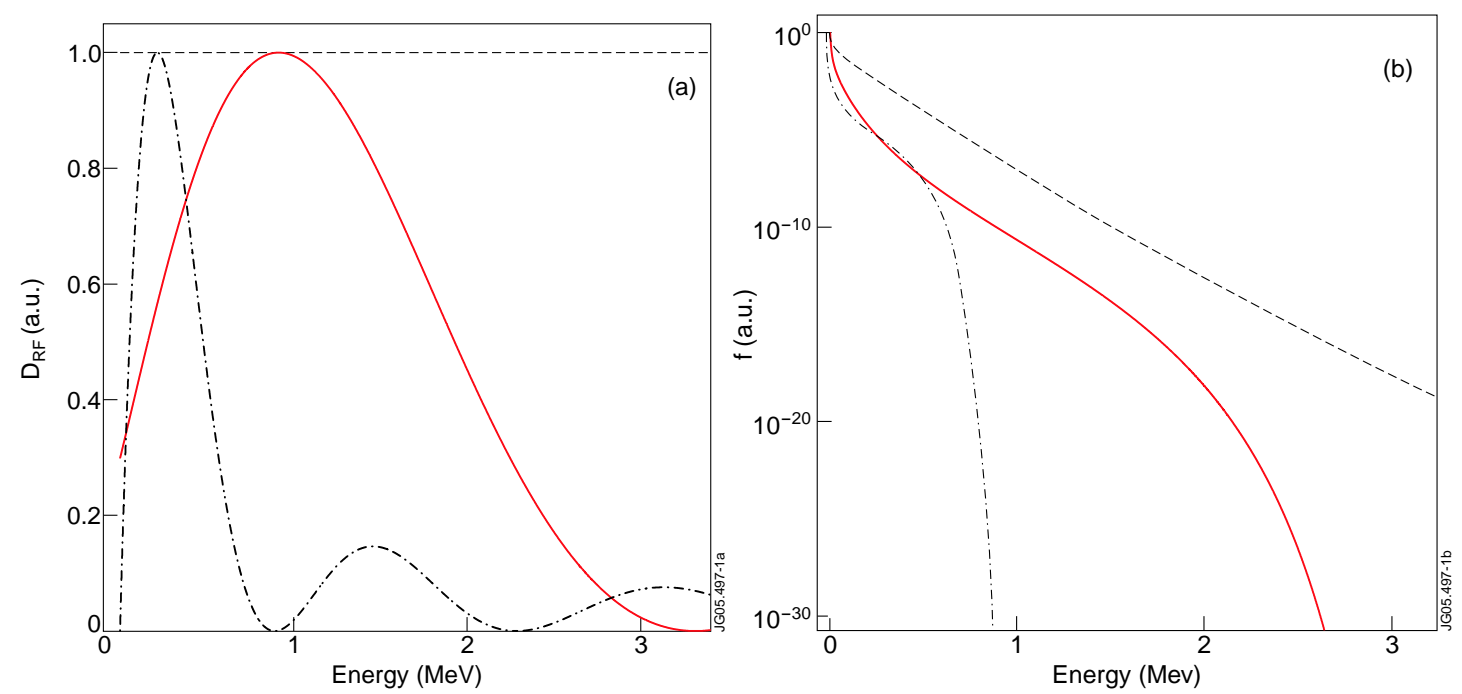

Figure 1. (a) Quasi linear RF diffusion coefficient for the fundamental (-) and second (- ·) harmonic ICRF heating. Dashed line (--) shows a fictitious case where $\mathrm{D}_{\mathrm{RF}}$ is constant. (b) The corresponding energy distributions calculated from Eq. (3).

Figs. 1(a) and 1(b) also demonstrate that already somewhat before $E^{*}$ values distributions start to clamp because the available power is not enough to compensate the slowing down of the ions. Figures make it also apparent why the second harmonic heating scheme was chosen for the experiments: $E^{*}$ value for the fundamental heating is typically at too high energies That is either particles experience orbit losses before reaching $\mathrm{E}^{*}$ or the available diagnostics capabilities on JET will not allow accurate measurements around $\mathrm{E}^{*}$ for fundamental heating.

For ICRF heating of minority ion species the ratio $\mathrm{E}_{-} / \mathrm{E}_{+}$stays almost constant as the electron density is varied. Consequently, in these cases the location of the first minimum of of $\mathrm{D}_{\mathrm{RF}}$ depends only on the argument of the Bessel functions, see Eq. (2). Writing $\alpha^{*}=k_{\perp} v_{\perp}^{*} / \omega_{c i}$, where $\alpha^{*}$. is the argument at the minimum and solving for $\mathrm{E}^{*}$ we get an approximate scaling $E^{*} \propto B^{2} / n_{e}$. Here, it was assumed that $\omega_{c i} \propto B$ and that $k_{\perp} \propto \sqrt{n_{e}}$ as given by from cold plasma theory

To show how electron density affects the distribution and its shape we have plotted three resonant particle energy distributions in Fig. 2 using parameters that are typical for the experiments performed. That is; second harmonic hydrogen minority heating in deuterium plasma with an electron density of $3 \cdot 10^{19} \mathrm{~m}^{-3}$ using dipole phasing of the JET ICRF antennas having the maximum power at $\mid \mathrm{k}_{\|} \|=8 \mathrm{~m}^{-1}$ and a frequency of 51 MHz. The broken lines symbolise cases where electron densities were varied keeping otherwise identical plasma parameters. PION code [4,5] was used to quantify the changes in the perpendicular wave number and in polarisation of the wave due to density changes. The graph shows that a ten percent change in the density results in a significant, and measurable, change in the distribution. We can now see that from the experimental point of view a convenient way of assessing the importance of FLR effects is to vary electron density (and thus $\mathrm{E}^{*}$ ) and see how well the distribution responds to it. 


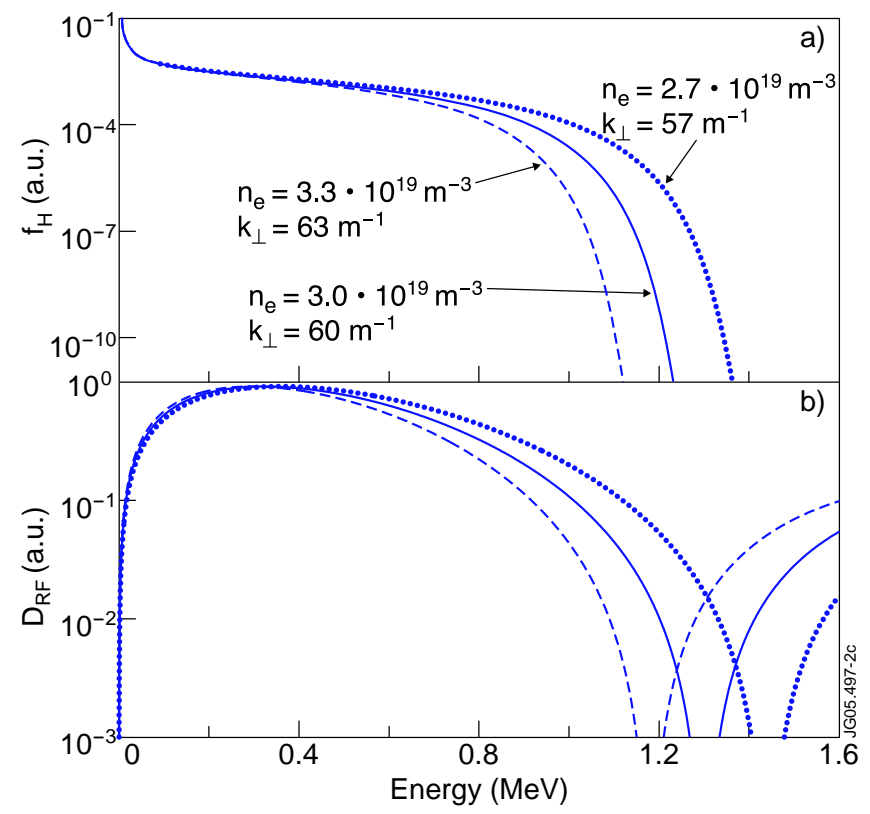

Figure 2. (a) Steady-state energy distribution functions of hydrogen with second-harmonic minority heating scenario, $(2 \mathrm{H}) \mathrm{D}$ and (b) the corresponding RF diffusion coefficients. Full lines are with ICR frequency $\mathrm{f}=51.25 \mathrm{MHz}$, electron temperature $\mathrm{T}_{\mathrm{e}}=4.5 \mathrm{keV}$, ion temperature $\mathrm{T}_{\mathrm{i}}=2.5 \mathrm{keV}$, electron density $\mathrm{n}_{\mathrm{e}}=3 \times 10^{19} \mathrm{~m}^{-3}$ and perpendicular wave number $\mathrm{k}_{\perp}=60 \mathrm{~m}^{-1}$. Broken lines correspond to a situation where $\mathrm{k}_{\perp}$ is altered by changing electron density by ten percent.

So far we have only considered monochromatic heating, i.e. a fast wave with a single wave number $k_{\perp}$. Of course, in reality a wave emitted from a finite antenna has a wave number spectrum. Having a $\mathrm{k}_{\perp}$-spectrum means that the effective $\mathrm{D}_{\mathrm{RF}}$ becomes an average over different $k_{\perp}$ and consequently $\mathrm{D}_{\mathrm{RF}}\left(\mathrm{E}^{*}\right)$ is not zero but has a finite value. It is not, however, feasible to incorporate all the necessary corrections due to the wave number spectrum or the effect of, e.g. non-constant plasma profiles, Doppler shift, non-Maxwellian particle distribution or orbit effects to the analytical equation. To do this, numerical simulations are required. Later, in Section 4 we will show simulation results where these effects are taken into account.

Thus from the previous considerations we can expect that the distribution drops rapidly in cases where the RF diffusion coefficient in the plasma has a pronounced first maximum and first minimum similar as shown in Figs. 1 and 2. Now taking a completely opposite point of view: if, for some reason, there were effects that would average out the RF diffusion coefficient to the extent that it could be approximated with a constant, the behaviour of the distribution would be markedly different. In that case the solution for Eq. (3) would have a nearly exponential form $f \approx f_{0} \exp \left(-E_{\perp} / T_{\text {eff }}\right)$ where $\mathrm{T}_{\text {eff }} \propto \mathrm{p}_{\perp} \mathrm{T}_{\mathrm{e}}^{3 / 2} / \mathrm{n}_{\mathrm{r}} \mathrm{n}_{\mathrm{e}}$ (see Ref. [2] and Fig. 1). Here $\mathrm{p}_{\perp}$ is the flux surface averaged power density absorbed by resonant species, $\mathrm{n}_{\mathrm{r}}$ is the density of the resonant ions and $T_{e}$ and $n_{e}$ are the temperature and density of the electrons, respectively. Hence, in the limit of constant $\mathrm{D}_{\mathrm{RF}}$, local ion tail temperature would increase with increasing power and electron temperature and decrease with increasing ion and electron density. As we will soon see this is not a good approximation and as it is well known Stix $T_{\text {eff }}$ approximation for fundamental heating [2] should not be used for second and higher harmonic heating cases. 
For completeness we mention here two additional effects which we have not yet discussed but which deserve some attention. The first one is sawtooth oscillations that were also present in the experiments reported in this paper. Many studies have shown that sawtooth oscillations redistribute energetic ions inside the sawtooth inversion radius, see e.g. a recent review in Ref. [6]. They are known to have the most pronounced effect on particles which are closely tied to a flux surface and thus feel the reconnection process. Secondly, redistribution favours particles that do not have a too small ratio of $\mathrm{v}_{\|} / \mathrm{v}_{\perp}$. This means that sawteeth are not expected to play a major role for the highly energetic ICRH accelerated particles both having a small $\mathrm{v}_{\|} / \mathrm{v}_{\perp}$ ratio and broad banana orbits.

Secondly, what needs to be discussed is the so-called super-adiabatic motion $[7,8]$ of ions. Super-adiabatic motion happens when the relative phase between the wave and the Larmor motion of the resonating ion is not sufficiently perturbed as required for true diffusion. When the phases become correlated ICRH does no longer heat the particles but they remain oscillating in phase space islands. Stochasticity of the phase results usually from particle collisions or from the non-linear interaction between the wave and particles. Normally, at energies in $\mathrm{MeV}$ range, collisions are not sufficient for randomising the phase and when in addition the wave-particle interaction becomes weak, as it does close to $\mathrm{E}^{*}$, the phase of the ion Larmor motion remains correlated with the wave, phase space islands are formed and particles are not heated to higher temperatures. For now we will disregard this effect, but we will return to it again in the Discussion.

\section{EXPERIMENTAL RESULTS}

The experiments reported here were designed and carried out on the JET tokamak to verify whether the details in the ICRH diffusion coefficient (i.e. FLR effects) have an effect on the high energy part of the resonant ion energy distribution or whether the distribution functions qualitatively obey the simple relation $T_{\text {eff }} \propto p_{\perp} T_{e}^{3 / 2} / n_{r} n_{e}$ as discussed in section 2. The scenario chosen for the experiments is second harmonic hydrogen minority heating in L-mode deuterium plasma, $(2 \mathrm{H}) \mathrm{D}$. This scenario is favourable because it allows $\mathrm{E}^{*}$ to be easily adjusted, by varying the plasma density, to be in the measurement window of the high-energy neutral particle analyser (NPA) [9] which was used to measure the high-energy ion distribution functions.

The eight energy channels of the NPA were set to measure the proton flux in the range of $0.29-1.1 \mathrm{MeV}$. The NPA has a vertical line-of-sight at major radius $3.07 \mathrm{~m}$, i.e. less than $10 \mathrm{~cm}$ to the low field side of the magnetic axis, and thus enables the deduction of the line integrated distribution function of fast ions close to the centre of plasma. The magnetic field in the experiment was $1.65 \mathrm{~T}$, the plasma current $1.65 \mathrm{MA}$ and the electron density was varied in the range $2.8 \times 10^{19}-4.4 \times 10^{19} \mathrm{~m}^{-3}$. Externally only ICRH (3-5 MW) was used for plasma heating. IC waves were launched with four JET A2 antennas applied with a frequency of $51.25 \mathrm{MHz}$ using dipole phasing (toroidal mode number at the maximum of the antenna power spectrum was $|\mathrm{N}| \approx 26$ ). In this configuration, the second harmonic hydrogen minority resonance locates close to the centre of the plasma. 


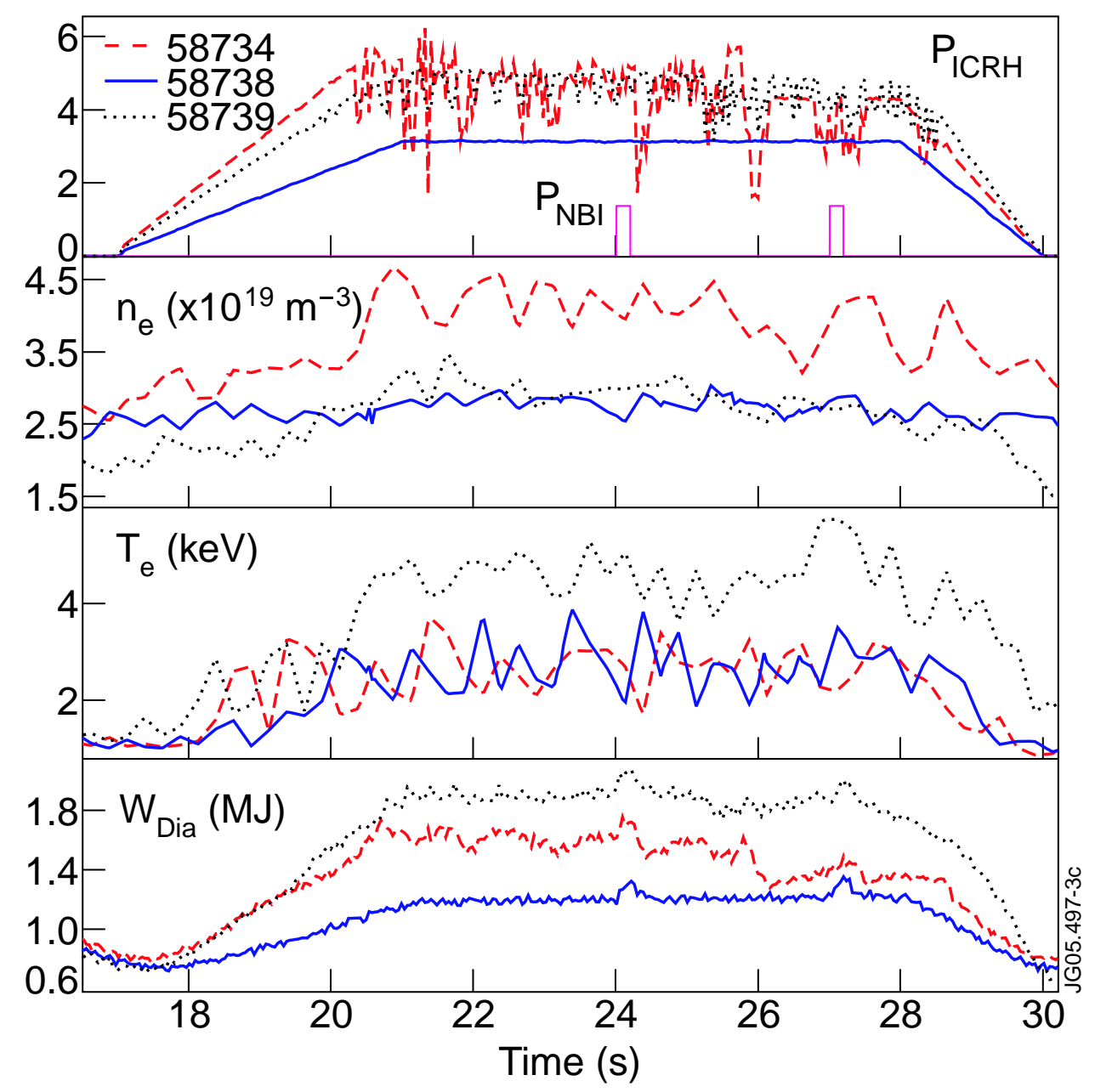

Figure 3. (a) ICRH and NBI power, (b) central electron density, (c) central electron temperature and (d) plasma diamagnetic energy. Charge exchange measurements from plasma centre at $\mathrm{t}=64.1 \mathrm{~s}$ gave for the effective charge of ions $Z_{\text {eff }}=1.75,1.45$ and 2.15 and for the ion temperature $\mathrm{T}_{\mathrm{i}}=1.2,1.2$ and $2.25 \mathrm{keV}$ for the pulses 58734,58738 and 58739 , respectively.

In Fig. 3 most relevant experimental parameters; ICRH power, neutral beam power (NBI), electron density and temperature at magnetic axis and plasma diamagnetic energy for the three discharges are shown. Short duration NBI pulses (blips) at 64.1s and at $67.1 \mathrm{~s}$ were used to measure the ion density and temperature. Plasma parameters in these discharges were such that initially 58738 and 58739 had identical plasmas (also identical electron densities) but due to higher input power electron temperature and plasma energy becomes higher in 58739. The plasma and power levels for discharge 58734 were chosen so that the effective temperature, $\mathrm{T}_{\text {eff }}$, is the same as in pulse 58738. The match was achieved by injecting more power in the high density pulse 58734. Table 1 highlights the relative differences for the most relevant parameters for these discharges. 
Table 1. The key parameters for the discharges. Values are normalised to those of discharge 58738 with an electron density $n_{e}(0)=2.8 \times 10^{19} \mathrm{~m}^{-3}$, power absorbed by hydrogen $P_{H}=2.9 \mathrm{MW}$, central electron temperature $\mathrm{T}_{\mathrm{e}}(0)=3 \mathrm{keV}$, hydrogen density $\mathrm{n}_{\mathrm{H}}(0)=1.3 \times 10^{18} \mathrm{~m}^{-3}$, effective tail temperature $\mathrm{T}_{\text {eff }}=1$ a.u. and $\mathrm{E}^{*}=1.37 \mathrm{MeV}\left(\mathrm{E}^{*}\right.$ has been evaluated in the plasma centre for $|\mathrm{N}|=26$, i.e. at the peak of the power spectrum).

\begin{tabular}{ccccccc}
\hline Pulse & $\mathrm{n}_{\mathrm{e} 0}$ & $\mathrm{P}_{\mathrm{H}}$ & $\mathrm{T}_{\mathrm{e} 0}$ & $\mathrm{n}_{\mathrm{H} 0}$ & $\mathrm{~T}_{\text {eff }}$ & $\mathrm{E}^{*}$ \\
\hline 58734 & 1.5 & 1.2 & 1.0 & 0.9 & 0.92 & 0.7 \\
58738 & 1.0 & 1.0 & 1.0 & 1.0 & 1.00 & 1.0 \\
58739 & 1.0 & 1.2 & 1.7 & 1.0 & 2.38 & 1.0 \\
\hline
\end{tabular}

The line integrated perpendicular energy distributions deduced from NPA measurements are shown in Fig. 4 for the three discharges of interest. From the comparison between discharges 58734 and 58738 one can see that the local tail temperature is significantly lower and the tail is shorter for the high electron density pulse 58734. At the same time $\mathrm{T}_{\text {eff }}$ is almost equal for both pulses and the location of first minimum in $\mathrm{D}_{\mathrm{RF}}, \mathrm{E}^{*}$, is about 30 percent smaller for the high density pulse 58734 (Table 1). These results indicate that $\mathrm{D}_{\mathrm{RF}}$ can not be considered as a constant. Otherwise both distributions should have the same shape and should not have a steep gradient region. Comparing the Table 1 and Fig. 4 we can see that $\mathrm{E}^{*}$ is reasonably accurate in estimating the energy at which the fast ion distribution rapidly decreases and effectively vanishes.
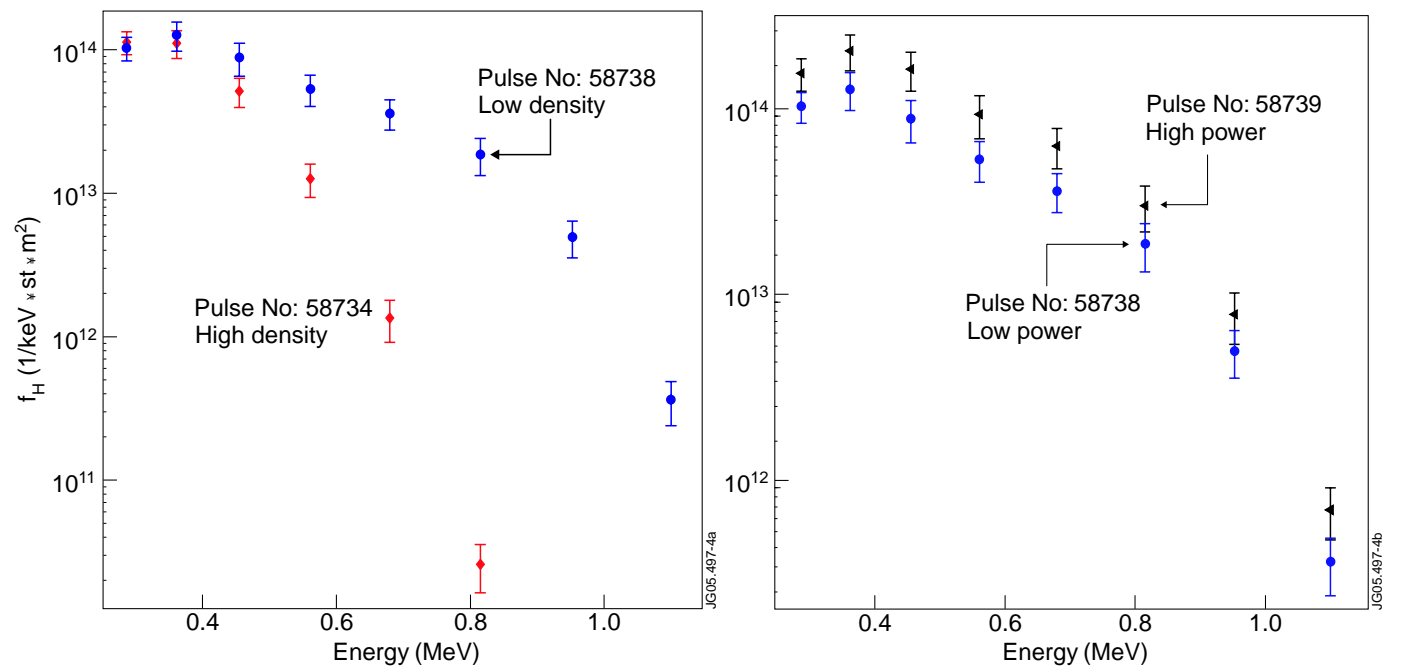

Figure 4. Experimental hydrogen energy distribution deduced from high-energy NPA measurements with error bars included.

The comparison between pulses 58738 and 58739 basically tells the same story from another viewpoint. This time the discharges have equal $\mathrm{E}^{*}$ but substantially different $\mathrm{T}_{\text {eff. }}$ In this case if FLR effects would not be important the discharge 58739 would have much higher temperature (Table 1). Experimental measurements, however, show identically shaped distributions (equal local temperatures) having the steeper gradient zones at the same place. Again, this provides clear evidence for the significance of FLR effects and rules out of $D_{R F}$ being a constant.

\section{COMPARISON WITH MODELLING}


For the modelling of the experiments ICRF codes PION [4] and FIDO [10] were used. PION is a simplified time-dependent Fokker-Planck code for calculating ICRF power deposition profiles and pitch-angle-averaged ion distribution functions selfconsistently (i.e. ICRF power deposition and the resulting resonant ion distributions are consistent). We used PION here to estimate the power absorbed directly on hydrogen as well as to solve the perpendicular wave number spectrum $\mathrm{k}_{\perp}$ and the polarisation of the wave. This data together with measured plasma profiles were used as input for the 3D Monte Carlo code FIDO to solve the full energy distribution of the resonant particles. The wave number spectrum fed into FIDO consists of ten perpendicular wave numbers. FIDO is a sophisticated code and can take e.g. the FLR effects, orbit effects, radial pinch effects and plasma profile effects into account. However, it does not handle sawtooth activity or super-adiabaticity which is why their possible additional effects have to be dealt separately, see Discussion. Other approaches for modelling this phenomenon could also be used. Possible choices include at least hot plasma theory, i.e. using a multi-harmonic asymptotic expansion of the wave driven effective electric field that accelerates the ions [11].

The comparisons between the simulated distributions and the measured ones are shown in Fig. 5. Solid lines show the calculated perpendicular energy distributions where simulations have been done using the measured plasma parameters. Dashed lines are from simulations made with a 10 percent higher/lower electron density while keeping ion fractions and other parameters constant. The data clearly show that simulations are consistent with the measurements with a rather good accuracy.

These simulations also confirm the sensitivity of the distribution on electron density as deduced from theory earlier. Since the uncertainty of measuring electron density at JET is normally 10 percent, we can take the regions between the dashed lines as an estimate for the uncertainty in modelling. Of course there are ambiguities also in other quantities such as in the excited $\mathrm{k}_{\|}$spectrum or the measured ones; ion densities and temperatures of different species etc. However, as explained in previous section the distribution is most sensitive to electron density variations which make this a sensible lower estimate for the total error. We can conclude that the uncertainties in measuring the plasma parameters alone are sufficient to explain the small differences. Unfortunately they also prevent one for making even more detailed comparisons between measurements and modelling and possibly finding out some minor details still missing from the simulations. 


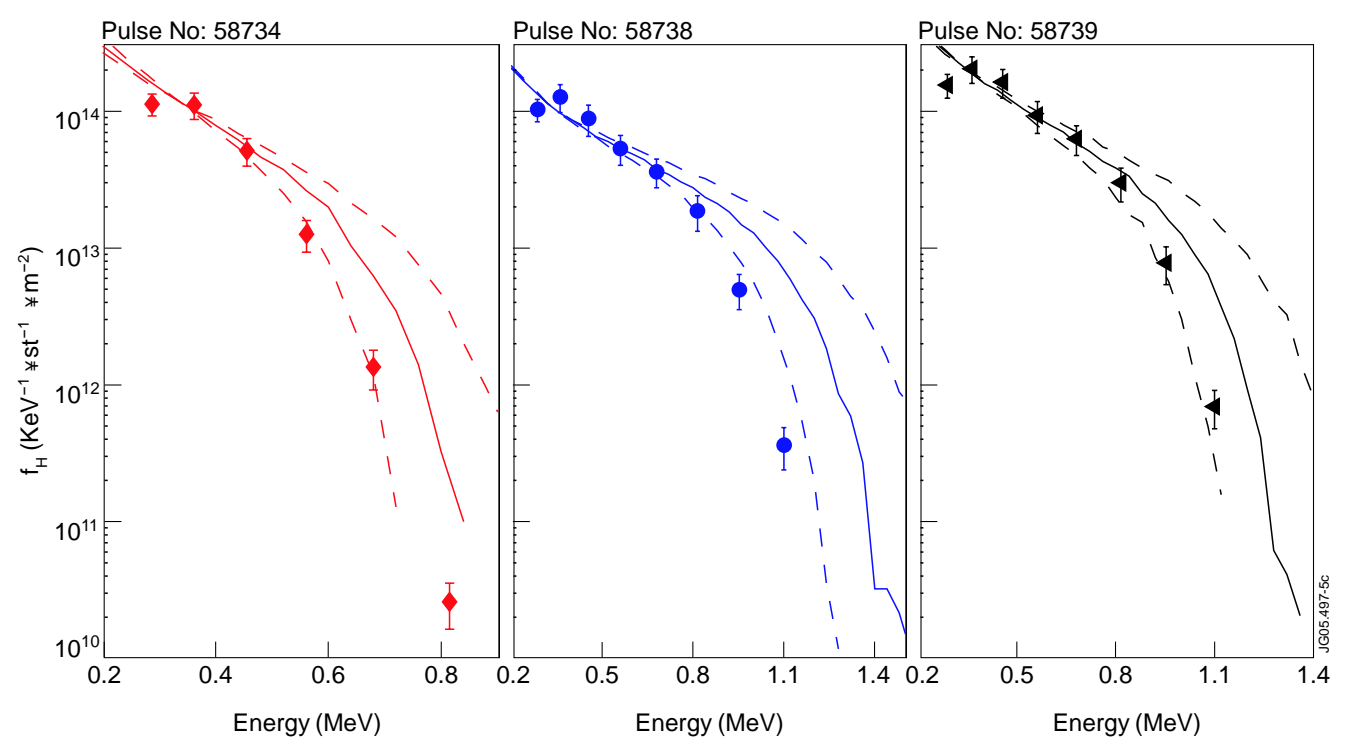

Figure 5. FIDO simulations of proton perpendicular energy distribution (lines) compared with NPA measurements (points with error bars). Dashed (--) lines reflect the uncertainty of modelling due to the uncertainty of plasma parameters.

\section{DISCUSSION}

Earlier, in section 2, we briefly discussed sawtooth activity in relation to fast ion distribution function. It was anticipated that they would not have a large effect on the highly energetic particles due to particles wide orbits and small $\mathrm{v}_{\|} / \mathrm{v}_{\perp}$ ratio. This is also supported by experimental data when we compare the sawtooth frequencies of the discharges. Approximately they were $6.5 \mathrm{~Hz}(58734), 7 \mathrm{~Hz}(58738)$ and $3.5 \mathrm{~Hz}$ (58739). Here, with about equal sawteeth frequencies discharges 58734 and 58738 have markedly distinct spectra and with quite different frequencies discharges 58738 and 58739 have similar spectra. Also, if the ion slowing down times of about $0.2 \mathrm{~s}$ (58734), 0.2s (58738) and 0.4s (58739) are taken into account we can not find a trend that would explain the measured perpendicular energy distributions.

The possible effect of the super-adiabatic motion was studied with a STOCH [7] code for this ICRF heating scenario. The results indeed indicated the presence of an adiabatic barrier just at the location of $\mathrm{E}^{*}$ for a monochromatic wave. Weak waveparticle interaction close to $\mathrm{E}^{*}$ can only create minor perturbations to the particle orbits leading to super-adiabatic motion which would tend to enhance the barrier created by the weak interaction (FLR effect) alone. We could not, however, obtain criteria for adiabacity in the presence of multiple frequencies and wave number spectra as would be required for a realistic case. The analysis of fundamental ICRH minority scheme [12] however shows that the inclusion of the spectral effects will increase stochasticity considerably and move the adiabatic barrier to higher energies. It was also seen that trapped ion motion is more stochastic than the motion of passing ions - here the energetic particles are mainly trapped. Adiabatic motion is therefore not likely to be of importance for the distribution below $\mathrm{E}^{*}$.

\section{CONCLUSIONS}


The experiments presented in this paper show clear clamping of the high-energy part of the second harmonic ICRF heated minority ions. The energy, around which the clamping occurs, is consistent with the expected energy where the ICRF waves and the particles interact weakly due to the finite Larmor radius effect. For the first time systematic experimental evidence together with modelling including relevant physics has been presented that demonstrates the importance of the finite Larmor radius effects on the high energy part of the resonant ion distribution. For all heating schemes, increased magnetic field will move $E^{*}$ to higher energies and increased electron density will move $\mathrm{E}^{*}$ to lower energies roughly as $E^{*} \propto B^{2} / n_{e}$. In ITER case the magnetic field and the electron density are normally significantly higher than here. Due to $E^{*}$ 's higher sensitivity on magnetic field strength $E^{*}$ will typically be in multi-MeV range in ITER. Thus, it can be concluded that the FLR effects are not likely to limit the fast ion energies in ITER. However, it also should be noted that the relatively low amount of power per particle available in ITER main heating scenarios will usually guarantee that the fast ion tail will not be able to develop up to $\mathrm{E}^{*}$ and therefore the ICRF power will be channelled to the bulk ions as desired.

\section{REFERENCES}

[1] M.J. Mantsinen, L-G. Eriksson, A. Gondhalekar, and T. Hellsten, Nuclear Fusion 39 (1999) 459.

[2] Stix, T.H., Nucl. Fusion 15, 737 (1975).

[3] Spitzer, L., Jr., The Physics of Fully Ionized Gases, $2^{\text {nd }}$ Revised Edition, Interscience, New York (1965).

[4] L.-G. Eriksson and T. Hellsten, Phys. Scripta 55, 70 (1995).

[5] L.-G. Eriksson, T. Hellsten, and U. Willén, Nucl. Fusion 33, 1037 (1993).

[6] Ya.I. Kolesnichenko, V.V. Lutsenko, R.B. White, Yu.V. Yakovenko, Nuclear Fusion, 40, 1325 (2000).

[7] Helander, P., Lisak, M., Phys. Fluids B 4, 1927 (1992).

[8] Stix, T.H., in Application of RF Waves to Tokamak Plasmas (Proc. Course and Workshop Varenna, 1985), Vol. 1, Monotypia Franchi, Perugia (1985) 24.

[9] A.A. Korotkov, A. Gondhalekar, and A.J. Stuart, Nucl. Fusion 37, 35 (1997).

[10] J. Carlsson, L.-G. Eriksson and T. Hellsten, Nuclear Fusion 32, 719 (1997).

[11] D.Testa, W.G.F.Core and A.Gondhalekar, Phys. Plasmas 6, 3498-3510 (1999).

[12] V. Bergeaud, F. Nguyen, A. Becoulet and L.-G. Eriksson, Phys Plasmas 8, 139 (2001). 